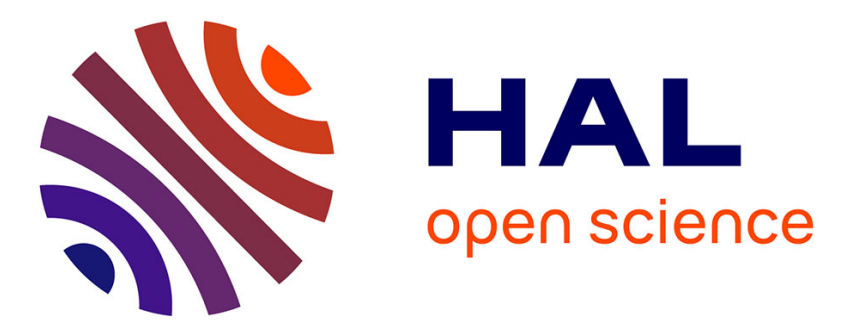

\title{
Fungal mediated innate immune memory, what have we learned?
}

Jessica Quintin

\section{To cite this version:}

Jessica Quintin. Fungal mediated innate immune memory, what have we learned?. Seminars in Cell and Developmental Biology, 2019, 89, pp.71-77. 10.1016/j.semcdb.2018.05.023 . pasteur-02873918

\section{HAL Id: pasteur-02873918 \\ https://hal-pasteur.archives-ouvertes.fr/pasteur-02873918}

Submitted on 23 Jun 2020

HAL is a multi-disciplinary open access archive for the deposit and dissemination of scientific research documents, whether they are published or not. The documents may come from teaching and research institutions in France or abroad, or from public or private research centers.
L'archive ouverte pluridisciplinaire HAL, est destinée au dépôt et à la diffusion de documents scientifiques de niveau recherche, publiés ou non, émanant des établissements d'enseignement et de recherche français ou étrangers, des laboratoires publics ou privés.

\section{다)(1) $(5$}

Distributed under a Creative Commons Attribution - NonCommercial| 4.0 International 


\section{Fungal mediated innate immune memory, what have we learned?}

Jessica Quintin ${ }^{\mathrm{a} \#}$

\section{Highlights}

- Innate immune cells can be trained by fungi and fungal components

- Innate immune training by fungi is mediated by metabolic shifts and epigenetic reprogramming

- Fungi and fungal components modify mammalian host response to fungal, bacterial, parasitic and viral infections

- Fungal components modify outcome of inflammatory disease and cancer

- Further studies on fungi/b-glucan induced innate immune memory are still required 


\section{Fungal mediated innate immune memory, what have we learned?}

Jessica Quintin $^{\mathrm{a}}$

${ }^{a}$ Immunology of Fungal Infections, Department of Mycology, Institut Pasteur, 25 rue du Docteur Roux, 75015, Paris, France. jessica.quintin@pasteur.fr

Corresponding author:

Dr. Jessica Quintin

Immunology of Fungal Infections

Mycology Department

Metchnikoff Building (67)

Institut Pasteur

25, rue du Docteur Roux

75015 Paris, France

Tel: +33 (0)144389454

Fax: +33 (0)1 40613115

Mail: jessica.quintin@pasteur.fr 


\begin{abstract}
The binary classification of mammalian immune memory is now obsolete. Innate immune cells carry memory characteristics. The overall capacity of innate immune cells to remember and alter their responses is referred as innate immune memory and the induction of a non-specific memory resulting in an enhanced immune status is termed "trained immunity". Historically, trained immunity was first described as triggered by the human fungal pathogen Candida albicans. Since, numerous studies have accumulated and deciphered the main characteristics of trained immunity mediated by fungi and fungal components.

This review aims at presenting the newly described aspect of memory in innate immunity with an emphasis on the historically fungal mediated one, covering the known molecular mechanisms associated with training. In addition, the review uncovers the numerous nonspecific effect that $\beta$-glucans trigger in the context of infectious diseases and septicaemia, inflammatory diseases and cancer.
\end{abstract}

Keywords: Innate immune memory, monocytes, fungi, $\beta$-glucan, disease 


\section{Introduction}

Humans are daily exposed to numerous potential pathogens present in the environment, through inhalation, ingestion and contact. Microorganisms cause disease only occasionally and our ability to prevent infection relies on our immune system, constituted by the adaptive and innate immune branches.

The adaptive immune system is very potent and remembers previous encounters with specific pathogens, destroys them upon additional attacks. Adaptive immune responses, however, are slow to develop. Specific T and B lymphocytes clones require to be activated and expand upon first exposure to a new pathogen; several days can pass before the responses are effective. Meanwhile, microbes will rapidly proliferate and spread to an advanced infection. During these first critical hours and days of exposure to a new pathogen, the host therefore relies on its innate immune system to protect itself from infection. Although innate immune responses are not antigen-specific to a particular pathogen as the adaptive immune responses are, they recognize highly conserved molecular patterns of pathogens through specific circulating or cellular receptors. Upon recognition, effective innate immune responses are mounted to fight and eradicate the invaders. Indeed, although innate immune responses in vertebrate are also able to activate the adaptive immune responses if necessary, most of the invading microorganisms are detected and destroyed within minutes or hours by the innate immune defence mechanisms. Innate immune responses are found in all metazoans whereas adaptive immune features only appeared 450 million in Gnathostomes. As such, plants and invertebrates only rely on the innate immune mechanisms and hence, are very successful in surviving infections and colonizing numerous varieties of niche, highlighting how potent innate immunity is. 
The objectives of this review are to present the newly described aspect of memory in innate immunity with an emphasis on the historically fungal mediated one, covering the known molecular mechanisms associated with this response. Finally, the review will present the numerous non-specific effects that $\beta$-glucans trigger.

\section{Innate immune memory}

\section{1. "trained" immunity and definition}

The mammalian immune system relies on two complementary branches, the innate and adaptive immunity. The classical and long-time accepted description of the immune system describes the innate immune system and associated effectors cells (monocytes, macrophages, neutrophils, and NK cells) as rapid, relatively non-specific, and unable to build immunological memory. On the other hand, the adaptive immune system takes longer to develop, is antigen-specific, and capable of immunological memory. Accumulating amount of past and recent studies highlight that innate immune cells can learn from previous encounter and alter their function [1]. This challenge in the classical paradigm is supported by a wide variety of reported data in plants, invertebrates, and mammals [2;3]. In mammals, cells of the innate immune compartments such as monocytes or natural killer (NK) cells, build up an innate memory upon a first challenge with certain microbes, pathogen-associated molecular patterns (PAMPs) or danger-associated molecular patterns (DAMPs). Once these cells encounter a secondary stimulus, either similar or unrelated to the first insult, their response is altered, which could result both in a stronger or attenuated response. The overall capacity of innate immune cells to remember and alter their responses is referred as innate immune memory [1] and the induction of a non-specific memory resulting in an enhanced immune status is termed "trained immunity" [3]. 
Trained immunity was demonstrated in mice in which T-/B-cell deficient animals are protected against a lethal fungal infection, with a significant reduction in mortality, when primed with a low dose of the human fungal pathogen Candida albicans [4]. Similar protection is mediated by the fungal cell wall component of the yeast, $\beta-(1-3,1-6)$ glucan [4].

C. albicans/ $\beta$-glucan mediated innate immune memory improves cellular host defence which ultimately leads to better survival of the host, a phenomenon requiring functional circulating monocytes [4]. The functional reprogramming of monocytes is induced through the $\beta$-glucan receptor dectin-1 and the non-canonical Raf-1 pathway, but not the Syk pathway [4] (Figure 1). $\beta$-glucan immune training of monocytes decreases ROS production-induced by zymosan, enhances microbicidal activities, and cytokine production in vivo and in vitro [4; 5].

\subsection{Molecular mechanisms underlying the induction of innate immune memory}

Innate immune memory is characterized by a complex interplay between immunological, metabolic and epigenetic changes. Gene repression or induction due to histone methylation depends on the position of methylation and the number of methyl groups (i.e., mono-, di-, or trimethylation). As such, trimethylation of histone $\mathrm{H} 3$ at lysine-4 (H3K4me3) at the promoter level is associated with gene activation, whereas H3K9me3 and H3K27me3 are repressive histone marks. Active enhancers are marked by mono- or dimethylation of H3K4. In addition, acetylation on lysine residues leads to the addition of negative charges to the positive lysines and thereby reduces the interaction between DNA and histones. As a result, the chromatin is less packed, increasing transcriptional accessibility at promoters or enhancers [6]. $\beta$-glucan mediated innate immune memory deeply modifies the epigenetic landscape of monocytes (Figure 1). With similarities observed in innate immune tolerance mediated by cecal ligation and puncture (CLP)s or lipopolysaccharide (LPS) [7], $\beta$-glucan affects the tri-methylation of 
the lysine 4 on the histone 3 at the promoter of numerous genes [4]. Increase of decrease in the H3K4me3 enrichment is correlated with transcriptional expression of the genes affected [4]. Interestingly and in contrast to innate immune tolerance, no effects are observed at the level of the dense heterochromatin mark H3K27me3 [4; 7]. However, the repressive histone H3K9me3 is associated with innate immune memory of both LPS tolerance and $\beta$-glucan training through the transcription factor ATF7 [8]. Latent enhancers are also observed in $\beta$-glucan trained monocytes, another aspect shared with LPS innate immune memory. It is therefore clear that innate immune memory (either trained immunity or tolerance) affect the epigenome of the host cell. Trained immunity and tolerance are both innate memory but immunologically opposed; could they also be mechanistically and epigenetically mirrored? Such mirror hypothesis would be supported by the fact that $\beta$-glucan can reverse epigenetic marks of LPS-tolerant cells [9]. However, although $\beta$-glucan and LPS affect both the epigenome, the genes affected are not all similar and shared. Actually, the specific signature induced by $\beta$-glucan is broader affecting a larger number of genes [8; 10].

Gene ontology analyses of the specific genes affected by $\beta$-glucan training of monocytes predicted the involvement of important immunological pathways and cellular metabolism. As such, cAMP-PKA-dependent signalling appears essential for innate immune memory activation by $\beta$-glucan and protection against infection [10] (Figure 1). $\beta$-glucan training generates in monocyte a shift from oxidative phosphorylation to aerobic glycolysis (the Warburg effect). The decrease in oxygen consumption and increase in lactate production and NAD+/NADH ratio require the Akt/mTOR/HIF-1 $\alpha$ [11] (Figure 1). Interestingly, not only glucose metabolism, but also other metabolic pathways play an important role in reprogramming and polarization of cells. Further analysis of the transcriptional signature of $\beta$-glucan trained monocytes demonstrates major differences observed in TCA cycle metabolites and fatty acid metabolism 
[12]. Glutaminolysis and accumulation of fumarate contribute to the trained immunity phenotype induced by $\beta$-glucan. Interestingly, these alterations in cellular metabolism influence the epigenetic program at the level of H3K4me3 and H3K27Ac of innate immune monocytes and by inhibiting KDM5 demethylases [12], placing metabolic pathways as crucial for maintenance and induction of innate immune memory (Figure 1). In addition, cholesterol synthesis pathway, but not the synthesis of cholesterol itself, modulates $\beta$-glucan trained immunity and epigenetic reprogramming. Induction of trained immunity by $\beta$-glucan depends on the intracellular mevalonate. The metabolite mevalonate itself recapitulates training obtained by $\beta$-glucan such as increased inflammation, H3K4me3 enrichment and lactate production via the activation of IGF1 receptor, and mTOR [13] (Figure 1).

$\beta$-glucan mediates increased inflammatory response and protection in mice against secondary challenges for several weeks [4]. Despite the short lifespan of monocytes in circulation, this protection relies on the presence of functional circulating monocytes, recruited in the periphery from the bone marrow through the chemokine receptor CCR2 [10]. The noticeable incompatibility between short lifespan of circulating monocytes and long-term protection could be explained by the fact that administration of $\beta$-glucan from Trametes versicolor $(\beta 1-4,1-3$, 1-6 branching) to mice affect the progenitors of the myeloid lineage and induces their expansion. The expansion of hematopoietic stem cells progenitors (HSPCs) (Figure 2) induced by $\beta$-glucan training correlates with a beneficial response to emergency myelopoiesis-induced by LPS and to chemotherapy-induced myelosuppression. $\beta$-glucan-induced myelopoiesis is associated with metabolic changes in glucose and lipid metabolism in the progenitors [14]. The effects of $\beta$-glucan on HPSCs are promoted by IL-1 $\beta$ signalling and associated with an enhanced activation of the GM-CSF/CD131 axis. Of interest, some of the effects on HSPCs, such as the increase in granulocyte macrophage progenitor (GMP) observed 7 days after $\beta$ - 
glucan administration, last for at least a month providing confidence that innate immune protection might persist for several weeks or even months.

\section{Innate immune memory in disease}

\subsection{Infection and septicaemia}

Traditional Chinese medicine has for centuries used fungi for healing and recent interests have focused on polysaccharides, that are a crucial component of fungi cell walls [15]. Within the diverse polysaccharides present in the cell wall, $\beta$-glucans are one of the key reason fungi are used in cosmetics, as food additives or as medicinal purposes [16]; they have also shown beneficial effects in the outcome of various diseases [17]. As such, administration of fungal $\beta$ glucans in infected animals as curative immunotherapy but, importantly, also before the onset of the infection (innate immune memory) can positively impact the evolution of a number of infectious diseases.

Monocytes and macrophages, main cells described to be affected by $\beta$-glucan training [4] play crucial role in the pathophysiology of sepsis and inflammation [18]. Sepsis, defined as a systemic host response to singular, or more often polymicrobial, infection, and associated with multiple organ dysfunction syndrome, remains one of the main cause of morbidity and mortality in intensive care units [19]. During the course of a severe systemic infection, recognition of bacterial components such as LPS by the innate immune cells trigger the release of mediators of inflammation that result in an overwhelming pathogenic inflammatory response in the circulation and vital organs. The proinflammatory cytokines TNF- $\alpha$, IL-1 and IL-6 are important mediators of sepsis and organ dysfunction [20]. In vitro $\beta$-glucan training of monocytes increases the inflammatory properties of the innate cells that are producing more TNF- $\alpha$, and IL-6 proinflammatory cytokines upon secondary stimulation with LPS. Therefore, 
one might hypothesize that fungal training will exacerbate the host immune responses and possibly the associated deleterious symptoms upon septicaemia. Supporting part of this hypothesis, during an endotoxin shock model in mice that mimics symptoms of septicaemia, training with $C$. albicans intravenous pre-injection increases the concentration of cytokines in circulation generated by the LPS infusion [4]. In contrast, however, the cell wall component $\beta$ glucans appears to be protective against multiple models of authentic septicaemia. Indeed, intraperitoneal injection of glucan 5 and 3 days prior to an Escherichia coli sepsis was already reported to be immunomodulatory 35 years ago [21]. Of interest, it is important to report that, opposed to the intraperitoneal route, intravenous injection of $\beta$-glucan is not protective and even deleterious [21]. Numerous complementary studies during the past years have confirmed the non-specific beneficial effect of intraperitoneal $\beta$-glucan pre-treatment of animals towards $E$. coli-induced experimental peritonitis and bacteraemia [22], involving hyperfunctional macrophages [23] and neutrophils dynamics [24]. Using a polymicrobial sepsis model with CLP, $\beta$-glucan pre-treatment generates early activation of $\mathrm{NF}-\kappa \mathrm{B}$ and nuclear factor interleukin 6 (NF-IL6)[25], down-regulation of IKK $\beta$ kinase activity and altered phosphorylation and degradation of $\mathrm{I} \kappa \mathrm{B} \alpha[26]$. $\beta$-glucan pre-treatment also stimulates the Phosphoinositide 3-Kinase pathway, which positively correlates with increased survival in septic mice [27]. Therefore, intraperitoneal administrated $\beta$-glucan confers protection against peritoneal E. coli, postoperative-C. albicans, S. aureus and polymicrobial CLP induced sepsis in mice [28; 29]. In addition, $\beta$-glucan shows beneficial protective properties in other mammals. As such, rats are protected by $\beta$-glucan intra-gastric prophylactic treatment against challenge with rat cecal contents [30] or against endotoxaemia induced by intravenous infusion of E. coli LPS [31]. The protection involves decrease in serum TNF- $\alpha$, increased GSH levels, decreased MDA levels and MPO activity, which diminish oxidative damage [32]. 
Finally, in humans, $\beta$-glucans are also capable to modulate ex vivo LPS-induced-tolerance in monocytes. Actually, ex vivo $\beta$-glucan exposure reinstates a responsive phenotype in both human monocytes tolerized by in vitro LPS exposure and monocytes tolerized by in vivo experimental endotoxaemia in healthy volunteers. This reversal of tolerance involves epigenomic reprogramming of macrophages [9].

C. albicans training induces protection in wild-type and T/B-cells deficient mice against a secondary $C$. albicans infection. Within $C$. albicans, $\beta$-glucan is the most-well known inducer of protective innate immune memory. Of interest, $\beta$-glucan from other yeast such as $S$. cerevisiae also triggers innate immune memory but with less effectiveness [33]. Chitin from a clinical S. cerevisiae strain also carries training abilities [33]. Actually, mice are protected against induced-candidiasis when intraperitoneally pre-treated with chitin or chitosan [34]. Such protective effect against candidiasis is also obtained with prophylactic intraperitoneal injection of S. cerevisiae-acidic mannans [35].

As highlighted above, protection induced by $C$. albicans, or its $\beta$-glucan cell wall component, is diverse and non-specific against both systemic candidiasis or peritoneal bacteraemia. In addition, $\beta$-glucan pre-treatment by intraperitoneal injection can also protect mice against intravenous inoculated systemic $S$. aureus infection $[11 ; 36]$. Incidence of $S$. aureus infection is predominant in patients with cancer and receiving immunotherapy. Interestingly, $\beta$-glucan pre-treatment can also confer protection to cyclophosphamide treated mice against $S$. aureus systemic infection [36]. The non-specific protection obtained by $\beta$-glucan against $S$. aureus septicaemia is also recapitulated in leukemic mice [28]. Not only systemic Staphylococcus infection show non-specific beneficial effect of $\beta$-glucan. Subcutaneous injection of glucan to ewes before lambing or during lactation allows protection against an induced S. haemolyticus mastitis up to 40 days after pre-treatment [37]. Mycobacterium bovis intravenous systemic 
infection in mice can also be controlled by $\beta$-glucan intravenous pre-treatment, with a dose dependent effect of $\beta$-glucan [38]. Similarly, $\beta$-glucan intraperitoneal pre-treatment triggers a non-specific antimicrobial effect in mice experimentally infected with Streptococcus pneumoniae [38]. In addition to fungal and bacterial infection, $\beta$-glucan is also potent in protecting animals against parasitic infection. $\beta$-glucan pre-treatment is effective against visceral Leishmaniasis in Hamsters [39] as well as against Leishmania major infection in mice [40]. Finally, virus-mediated diseases seem also modulated by $\beta$-glucan. The survival time of mice to hepatitis virus strain MHV-A59 is significantly prolonged upon $\beta$-glucan intravenous pre-treatment, although maximum effectiveness is reached when glucan is administrated both before and after the viral challenge [41]. Similarly, pneumonia induced by swine influenza virus is significantly less severe in orally pre-administered $\beta$-glucan animals, with less lung lesions and more IFN-ץ and nitric oxide in bronchoalveolar lavage fluid [42]. Altogether, local and systemic $\beta$-glucans pre-treatment enhances immune protection of diverse mammalian host from mice to rats and monkeys, towards non-specific infectious diseases including viruses, bacteria and fungi inoculated through diverse primary infecting routes (intravenous, subcutaneous, intranasal etc....)[43].

\subsection{Inflammatory disease and cancer}

Inflammation that are caused by pathogens, can also lead to damage of cells and tissues. Inflammatory bowel disease is a chronic idiopathic inflammatory disease of the gastrointestinal tract affecting significant population numbers in the Western world. Chronic inflammation in inflammatory bowel disease, such as ulcerative colitis and Crohn's Disease, is characterized by the distortion of the intestinal epithelium structure and repeated episode of impaired mucosal barrier functions permitting the infiltration of immune inflammatory cells. In this inflammatory background, one might hypothesize that fungi or $\beta$-glucan would exacerbate the inflammation. 
However, literature in the field does not permit to corroborate or invalidate the hypothesis. Oral administration of $\beta$-glucan from eatable mushrooms or yeast reduces the acute inflammation in the ulcerative colitis triggered by dextran sulfate sodium (DSS) [44; 45]. Protection against DSS-induced colitis includes reduced clinical symptoms (body weight loss), reduced inflammatory cells infiltration and cell apoptosis in the colonic tissue, and ameliorated intestinal permeability. Moreover, treatment with $\beta$-glucan decreases the concentration of malondialdehyde (MDA), myeloperoxidase (MPO), the levels of eosinophil peroxidase and Nacetyl- $\beta$-D-glucosaminidase, but also inhibits the expression of iNOS and several inflammatory factors: TNF- $\alpha$, IL-1 $\beta$ and IL-6 as well as nitric oxide (NO) of the colonic tissues [44; 45]. In total contrast, one other study highlights that mice orally pre-treated with $\beta$-glucans (either with curdlan (a particulate $\beta$-glucan), with glucan phosphate (a soluble $\beta$-glucan), or with zymosan (particle made from S. cerevisiae, which contains around $55 \% \beta$-glucans)) for 2 weeks present a rather similar or even aggravated DSS-induced intestinal inflammation [46]. Weight loss, colon weight, and faeces scores did not differ and $\beta$-glucan-treated mice have increased inflammation at the histological level. Furthermore, curdlan and zymosan, but not soluble glucan treatment leads to increased inflammatory cytokines and chemokines in the colon. The discrepancies between this latter study and the three former on the beneficial effect of $\beta$-glucan in colitis might rely in the amount of glucan administrated, the source and branching/structure of $\beta$-glucan and more importantly the solubility. In fact, the pharmacokinetics following intravenous administration of three different highly purified and previously characterized $\beta$ glucans were studied using carbohydrates covalently labelled with a fluorophore on the reducing terminus. The variations in molecular size, branching frequency and solution conformation have an impact on the elimination half-life, volume of distribution and clearance [47]. 
Autoimmune inflammatory diseases are often multi-factorial. As such a combination of genetic and environmental factors can cause autoimmune disease in animals. SKG mice, which are genetically prone to develop autoimmune disease, fail to develop arthritis in the absence of any microbes. In this sterile context, $\beta$-glucan injection triggers the severe chronic arthritis in mice [48]. In addition, $\beta$-glucans also trigger spondyloarthritis and crohn's disease-like ileitis in SKG Mice [49]. Of note, in vitro training of monocytes from healthy volunteers renders them more potent in forming foam cells, one of the deleterious macrophages phenotype in atherosclerosis [50]. Therefore, although one cannot formally relate the effects to innate immune memory, fungi and $\beta$-glucans alone can induce autoimmune inflammation by stimulating innate immunity in individuals who bear genetic anomalies or variations. Despite these reports on the putative negative effects of $\beta$-glucan training in the induction of inflammatory disorders affecting the arterial vessels, other studies encourage the use of $\beta$ glucan in the treatment of diabetes and associated cardiovascular risks [51]. Dietary intake of $\beta$-glucans is known to reduce the risk factors and complications associated with diabetes such as vascular injury leading to heart disease (due to decreased glucose transportation from blood into muscle and fat cells). More specifically, fungal $\beta$-glucans reduce blood glucose concentrations after oral administration in animal experiments and in clinical trials, it reduces blood cholesterol and hypertension. In addition, $\beta$-glucans also promote wound healing and alleviate ischemic heart injury (reviewed in details in [51]). However, again, one cannot firmly associate the mechanisms behind the effect of $\beta$-glucans on diabetes and associated complications with innate immune memory. Altogether, $\beta$-glucan seems to have controversial effects on inflammatory disease.

Cancer outcome improvement has long been associated with $\beta$-glucan administration after the onset of the disease and tumour formation [52; 53]. $\beta$-Glucans are immunomodulators with 
beneficial properties in cancer immunotherapy and recent efforts towards optimizing tumour microenvironment suggest the use of $\beta$-glucan-based nanoparticles treatment [54]. Although opsonized with iC3b (recognized by CD18 on leukocytes), tumour cells do not activate leukocytes for complement receptor 3 (CR3)-dependent cellular cytotoxicity. This natural resistance of iC3b-opsonized-tumor cells rely on the lack of ligand to activate CD11b. Soluble yeast $\beta$-glucan (ligand for CD11b) override the normal resistance of iC3b-postive tumour cells, allowing this important cytotoxic effector mechanism of the complement system to function against tumour cells. Cytotoxic activation of the CR3 of $\beta$-glucan-primed-NK cell by iC3bopsonized tumours is shown to be accompanied by a tumour-localized secretion of the cytokines TNF- $\alpha$, IFN- $\alpha$, IFN- $\gamma$, and IL-6 [55; 56]. $\beta$-Glucans are also synergetic boosters of bioactive molecules such as resveratrol, vitamin $\mathrm{C}$ or anti-tumour mAbs [57; 58]. Compared to the individual components, the combination of $\beta$-glucan with resveratrol and vitamin $C$ is the strongest activator of phagocytosis and antibody formation and strongly suppressed the growth of breast and lung tumours [57; 58]. Similarly, combined treatment with mAb plus $\beta$-glucan produces significantly greater tumour regression in several tumour model in mice [58]. These data suggest that the therapeutic efficacy of mAbs known to activate complement (e.g., Herceptin, Rituxan, and Erbitux) could be significantly enhanced if they are combined with $\beta$ glucan. Interestingly, tumoricidal activities are mediated with both intravenous administration of soluble $\beta$-glucan from $S$. cerevisiae or oral administration of WGP from Barley $\beta$-1,3-glucan and requires functional granulocytes [58; 59].

Although $\beta$-glucan seems very promising as a curative treatment of cancer with tumour regression these effects cannot be firmly associated with innate immune memory as treatment occur after the onset of the disease. So, what about $\beta$-glucan innate immune memory in preventing cancer? Interestingly, prophylactic intravenous administration of $\beta$-glucan purified from S. cerevisiae significantly inhibits lung metastasis triggered by the injection of colon 
carcinoma or melanoma cells. The inhibition is dose-dependent [60]. $\beta$-glucan also significantly prevents metastasis in liver and spleen produced by intravenous inoculation of lymphoma cells in mice [60]. Furthermore, prophylactic $\beta$-glucan treatment before tumour inoculation significantly prolongs the survival time of tumour-bearing mice. $\beta$-glucan seems to inhibit tumour metastasis via activation of macrophages and NK cells [60].

\section{Conclusions and Perspectives}

With recent discoveries highlighted in this review, the claim that innate immune cells and responses are devoid of any memory is now invalid. Innate immune cells can recall a first encounter and respond differently to the same pathogen or to an unrelated insult. Fungi and fungal related cell wall polysaccharides $\beta$-glucans are potent immune regulators. They are capable of inducing innate immune memory in monocytic phagocytes mediated by metabolism shifts, imprinting a specific epigenetic signature. Innate immune memory imprinted by fungi and fungal cell wall components triggers training against numerous and diverse secondary encounters ranging from gram-positive, gram-negative bacteria, fungi, parasites and viruses. It might also affect responses to numerous chronic inflammatory diseases as well as cancer.

Eatable mushrooms have been used for several thousands of years, back to the ancient Egyptians and ancient Chinese cultures, for countless illnesses and to promote general health and longevity. Therefore, one might conclude that mushrooms and fungal components such as $\beta$-glucan, via innate immune training, trigger only pleiotropic non-specific beneficial effects. However, few studies have been mentioned in this review in which $\beta$-glucan/fungi could be deleterious. Each of this potential adverse effect of $\beta$-glucan can be discussed. A first example is the detrimental capacity of fungi, as a microbe, to trigger inflammatory disease in genetically susceptible host. This effect is specific to SKG mice, which bear a point mutation in the ZAP- 
70 gene and spontaneously develop Th17 cell dependent arthritis in a conventional facility [61]. When reared in a specific pathogen free facility, SKG mice remain healthy until they are exposed to environmental agents such as fungi that are potent inducer of the Th17 response [48]. Other non-fungal related microbes and Th17 inducers would recapitulate the trigger of the disease. A second point of debate concerns $\beta$-glucan in infectious disease, which appears to be protective against multiple models of septicaemia. The few discrepancies observed in their protective effects are certainly due to the route of infection. As such a certain pattern appears in which direct intravenous inoculation of the training/protective molecules are less effective if not deleterious [21]. This is also intriguingly linked to the capacity of the training molecules to regulate cytokines. Only oral treatment with glucan attenuates the LPS-induced rise in plasma creatinine levels during induced-septicaemia [62] whereas a moderate baseline increase in IL$1 \beta$ levels is only observed in the glucan sub-cutaneous treated group. This discrepancy in protection is associated with a significant increase in IL-10 in the glucan sub-cutaneous treated group only, and with a significant decrease in IL-2 in the glucan orally treated group only [62]. Therefore, it seems that the local peritoneal or oral route is the beneficial glucan training route to privilege.

Finally, although some of the reported effects cannot be firmly associated with innate immune memory, the studies presented in this review use fungi and cell wall component as prophylaxis. It is important to acknowledge that $\beta$-glucan is also very potent when inoculated after the onset of the infection, inflammatory disease, or cancer $[38 ; 63 ; 64]$. Of note, the quality of the protection obtained after the onset of the septicaemia in rats and mice might be gender-specific, with a longer protection mediated in female mice $[63 ; 64]$.

Naturally, and important to acknowledge as well, $\beta$-glucan with its inherent capacity to train monocytic cell lineage represents also a potent adjuvant in vaccine triggering adaptive immune memory or in adjunctive therapy [65; 66] 
Altogether, despite the recent discoveries of the past decade and because of the few discrepancies reported, research should continue to focus on the exact mechanisms triggered by fungi and related components during innate immune memory, in order to safely benefit in the future from all the broad potential of trained immunity.

\section{$\underline{\text { Declaration of interest }}$}

The author declares no financial or commercial conflict of interest.

\section{Acknowledgments}

I thank Dr. Giorgio Camilli for the help during research and discussions. This work was supported by the ANR JCJC grant [ANR-16-CE15-0014-01] and the Institut Carnot Pasteur MI grant [ANR 11-CARN 017-01] (to J.Q.).

\section{Figures}

Figure 1:

Induction of trained immunity (innate immune memory) through recognition of $\beta 1-3,1-6$ glucans by dectin-1 is mediated by activation of immune and metabolic pathways which result in epigenetic rewiring of cellular functional programs in monocytes. Raf-1 and cAMP-PKAdependent signalling are important mechanisms of trained immunity activation. The dramatic changes in the epigenetic landscape is observed at the level of histone methylation and acetylation of both promoters and enhancers. The important rewiring of cellular metabolism includes a shift toward glycolysis induced through the mTOR pathway, as well as glutaminolysis and cholesterol synthesis. Specifically, accumulation of fumarate, due to glutamine replenishment of the TCA cycle, integrates immune and metabolic circuits to induce 
monocyte epigenetic reprogramming by inhibiting KDM5 histone demethylases. The metabolite mevalonate mediates training via activation of IGF1-R and mTOR and subsequent histone modifications in inflammatory pathways Induction of trained immunity through recognition of $\beta$-glucan by dectin-1 culminates into increased cytokine secretion, greater inflammatory response and enhanced protection towards non-specific secondary infections. mTOR, mammalian target of rapamycin; HIF-1 $\alpha$, hypoxia-inducible factor $1 \alpha$; TCA cycle, tricarboxylic acid cycle; KDM, lysine demethylase

\section{Figure 2}

Trained immunity and the modulation of hematopoietic stem and progenitor cells (HSPCs). Administration of $\beta$-glucan (prototypical trained-immunity-inducing agonist) to mice is recognized by unknown cells and triggers an increase concentration of IL-1 $\beta$ and granulocytemacrophage colony-stimulating factor (GM-CSF) in the bone marrow extracellular fluid. Administration of $\beta$-glucan also drives expansion of HSPCs subpopulation with variations over time (hematopoietic progenitors (LSKs; $\mathrm{Lin}^{-} \mathrm{CKit}^{+} \mathrm{Sca} 1^{+}$), multipotent progenitors (MPPs), long-term hematopoietic stem cells (LT-HSCs), short-term (ST)-HSCs). The effects of $\beta$ glucan on HPSC are promoted by IL-1 $\beta$ signalling. Adaptations in glucose metabolism and cholesterol biosynthesis is observed in LT-HSCs and increase in cell cycle markers is noticed in myeloid-based CD41+ LT-HSCs. The increase in myelopoiesis is associated with a beneficial response to DNA damage induced by secondary LPS challenge and protection from chemotherapy-induced myelosuppression in mice. 


\section{References}

[1] M.A. Hamon, and J. Quintin, Innate immune memory in mammals. Semin Immunol (2016).

[2] B. Milutinovic, and J. Kurtz, Immune memory in invertebrates. Semin Immunol 28 (2016) 328-42.

[3] M.G. Netea, J. Quintin, and J.W. van der Meer, Trained immunity: a memory for innate host defense. Cell Host Microbe 9 (2011) 355-61.

[4] J. Quintin, S. Saeed, J.H. Martens, E.J. Giamarellos-Bourboulis, D.C. Ifrim, C. Logie, L. Jacobs, T. Jansen, B.J. Kullberg, C. Wijmenga, L.A. Joosten, R.J. Xavier, J.W. van der Meer, H.G. Stunnenberg, and M.G. Netea, Candida albicans infection affords protection against reinfection via functional reprogramming of monocytes. Cell Host Microbe 12 (2012) 223-32.

[5] S. Bekkering, B.A. Blok, L.A. Joosten, N.P. Riksen, R. van Crevel, and M.G. Netea, In Vitro Experimental Model of Trained Innate Immunity in Human Primary Monocytes. Clinical and vaccine immunology : CVI 23 (2016) 926-933.

[6] M.A. Hoeksema, and M.P. de Winther, Epigenetic Regulation of Monocyte and Macrophage Function. Antioxid Redox Signal 25 (2016) 758-774.

[7] H. Wen, Y. Dou, C.M. Hogaboam, and S.L. Kunkel, Epigenetic regulation of dendritic cellderived interleukin-12 facilitates immunosuppression after a severe innate immune response. Blood 111 (2008) 1797-804.

[8] K. Yoshida, T. Maekawa, Y. Zhu, C. Renard-Guillet, B. Chatton, K. Inoue, T. Uchiyama, K. Ishibashi, T. Yamada, N. Ohno, K. Shirahige, M. Okada-Hatakeyama, and S. Ishii, The transcription factor ATF7 mediates lipopolysaccharide-induced epigenetic changes in macrophages involved in innate immunological memory. Nat Immunol 16 (2015) 1034-43.

[9] B. Novakovic, E. Habibi, S.Y. Wang, R.J.W. Arts, R. Davar, W. Megchelenbrink, B. Kim, T. Kuznetsova, M. Kox, J. Zwaag, F. Matarese, S.J. van Heeringen, E.M. JanssenMegens, N. Sharifi, C. Wang, F. Keramati, V. Schoonenberg, P. Flicek, L. Clarke, P. Pickkers, S. Heath, I. Gut, M.G. Netea, J.H.A. Martens, C. Logie, and H.G. Stunnenberg, beta-Glucan Reverses the Epigenetic State of LPS-Induced Immunological Tolerance. Cell 167 (2016) 1354-1368 e14.

[10] S. Saeed, J. Quintin, H.H. Kerstens, N.A. Rao, A. Aghajanirefah, F. Matarese, S.C. Cheng, J. Ratter, K. Berentsen, M.A. van der Ent, N. Sharifi, E.M. Janssen-Megens, M. Ter Huurne, A. Mandoli, T. van Schaik, A. Ng, F. Burden, K. Downes, M. Frontini, V. Kumar, E.J. Giamarellos-Bourboulis, W.H. Ouwehand, J.W. van der Meer, L.A. Joosten, C. Wijmenga, J.H. Martens, R.J. Xavier, C. Logie, M.G. Netea, and H.G. Stunnenberg, Epigenetic programming of monocyte-to-macrophage differentiation and trained innate immunity. Science 345 (2014) 1251086.

[11] S.C. Cheng, J. Quintin, R.A. Cramer, K.M. Shepardson, S. Saeed, V. Kumar, E.J. Giamarellos-Bourboulis, J.H. Martens, N.A. Rao, A. Aghajanirefah, G.R. Manjeri, Y. Li, D.C. Ifrim, R.J. Arts, B.M. van der Meer, P.M. Deen, C. Logie, L.A. O'Neill, P. Willems, F.L. van de Veerdonk, J.W. van der Meer, A. Ng, L.A. Joosten, C. Wijmenga, H.G. Stunnenberg, R.J. Xavier, and M.G. Netea, mTOR- and HIF-1alpha-mediated aerobic glycolysis as metabolic basis for trained immunity. Science 345 (2014) 1250684.

[12] R.J. Arts, B. Novakovic, R. Ter Horst, A. Carvalho, S. Bekkering, E. Lachmandas, F. Rodrigues, R. Silvestre, S.C. Cheng, S.Y. Wang, E. Habibi, L.G. Goncalves, I. Mesquita, C. Cunha, A. van Laarhoven, F.L. van de Veerdonk, D.L. Williams, J.W. van der Meer, C. Logie, L.A. O'Neill, C.A. Dinarello, N.P. Riksen, R. van Crevel, C. Clish, R.A. Notebaart, L.A. Joosten, H.G. Stunnenberg, R.J. Xavier, and M.G. Netea, 
Glutaminolysis and Fumarate Accumulation Integrate Immunometabolic and Epigenetic Programs in Trained Immunity. Cell Metab 24 (2016) 807-819.

[13] S. Bekkering, R.J.W. Arts, B. Novakovic, I. Kourtzelis, C. van der Heijden, Y. Li, C.D. Popa, R. Ter Horst, J. van Tuijl, R.T. Netea-Maier, F.L. van de Veerdonk, T. Chavakis, L.A.B. Joosten, J.W.M. van der Meer, H. Stunnenberg, N.P. Riksen, and M.G. Netea, Metabolic Induction of Trained Immunity through the Mevalonate Pathway. Cell 172 (2018) 135-146 e9.

[14] I. Mitroulis, K. Ruppova, B. Wang, L.S. Chen, M. Grzybek, T. Grinenko, A. Eugster, M. Troullinaki, A. Palladini, I. Kourtzelis, A. Chatzigeorgiou, A. Schlitzer, M. Beyer, L.A.B. Joosten, B. Isermann, M. Lesche, A. Petzold, K. Simons, I. Henry, A. Dahl, J.L. Schultze, B. Wielockx, N. Zamboni, P. Mirtschink, U. Coskun, G. Hajishengallis, M.G. Netea, and T. Chavakis, Modulation of Myelopoiesis Progenitors Is an Integral Component of Trained Immunity. Cell 172 (2018) 147-161 e12.

[15] R. Chang, Bioactive polysaccharides from traditional Chinese medicine herbs as anticancer adjuvants. J Altern Complement Med 8 (2002) 559-65.

[16] O. Rop, J. Mlcek, and T. Jurikova, Beta-glucans in higher fungi and their health effects. Nutr Rev 67 (2009) 624-31.

[17] R.P. Pelley, and F.M. Strickland, Plants, polysaccharides, and the treatment and prevention of neoplasia. Crit Rev Oncog 11 (2000) 189-225.

[18] S.K. Biswas, and E. Lopez-Collazo, Endotoxin tolerance: new mechanisms, molecules and clinical significance. Trends Immunol 30 (2009) 475-87.

[19] D.C. Angus, W.T. Linde-Zwirble, J. Lidicker, G. Clermont, J. Carcillo, and M.R. Pinsky, Epidemiology of severe sepsis in the United States: analysis of incidence, outcome, and associated costs of care. Crit Care Med 29 (2001) 1303-10.

[20] D. Annane, E. Bellissant, and J.M. Cavaillon, Septic shock. Lancet 365 (2005) 63-78.

[21] D.L. Williams, W. Browder, R. McNamee, and N.R. Di Luzio, Glucan immunomodulation in experimental E. coli sepsis. Adv Exp Med Biol 155 (1982) 701-6.

[22] E.T. O., O. Busayo, and O.J. K., The protective effect of beta glucan against Escherichia coli infected mice via intraperitonial administration. Journal of Bacteriology Research Vol. 3(2) (2011) pp.28-31.

[23] D.L. Williams, I.W. Browder, and N.R. Di Luzio, Immunotherapeutic modification of Escherichia coli--induced experimental peritonitis and bacteremia by glucan. Surgery 93 (1983) 448-54.

[24] D.L. Williams, E.R. Sherwood, I.W. Browder, R.B. McNamee, E.L. Jones, J. Rakinic, and N.R. Di Luzio, Effect of glucan on neutrophil dynamics and immune function in Escherichia coli peritonitis. J Surg Res 44 (1988) 54-61.

[25] D.L. Williams, T. Ha, C. Li, J.H. Kalbfleisch, J.J. Laffan, and D.A. Ferguson, Inhibiting early activation of tissue nuclear factor-kappa B and nuclear factor interleukin 6 with (1-->3)-beta-D-glucan increases long-term survival in polymicrobial sepsis. Surgery 126 (1999) 54-65.

[26] D.L. Williams, T. Ha, C. Li, J. Laffan, J. Kalbfleisch, and W. Browder, Inhibition of LPSinduced NFkappaB activation by a glucan ligand involves down-regulation of IKKbeta kinase activity and altered phosphorylation and degradation of IkappaBalpha. Shock 13 (2000) 446-52.

[27] D.L. Williams, C. Li, T. Ha, T. Ozment-Skelton, J.H. Kalbfleisch, J. Preiszner, L. Brooks, K. Breuel, and J.B. Schweitzer, Modulation of the phosphoinositide 3-kinase pathway alters innate resistance to polymicrobial sepsis. J Immunol 172 (2004) 449-56.

[28] N.R. Di Luzio, and D.L. Williams, Protective effect of glucan against systemic Staphylococcus aureus septicemia in normal and leukemic mice. Infect Immun 20 (1978) 804-10. 
[29] I.W. Browder, D.L. Williams, A. Kitahama, and N.R. Di Luzio, Modification of postoperative C. albicans sepsis by glucan immunostimulation. Int J Immunopharmacol 6 (1984) 19-26.

[30] A.O. Tzianabos, F.C. Gibson, 3rd, R.L. Cisneros, and D.L. Kasper, Protection against experimental intraabdominal sepsis by two polysaccharide immunomodulators. J Infect Dis 178 (1998) 200-6.

[31] A. Sandvik, Y.Y. Wang, H.C. Morton, A.O. Aasen, J.E. Wang, and F.E. Johansen, Oral and systemic administration of beta-glucan protects against lipopolysaccharide-induced shock and organ injury in rats. Clin Exp Immunol 148 (2007) 168-77.

[32] G. Sener, H. Toklu, F. Ercan, and G. Erkanli, Protective effect of beta-glucan against oxidative organ injury in a rat model of sepsis. Int Immunopharmacol 5 (2005) 138796.

[33] L. Rizzetto, D.C. Ifrim, S. Moretti, N. Tocci, S.C. Cheng, J. Quintin, G. Renga, V. Oikonomou, C. De Filippo, T. Weil, B.A. Blok, M.S. Lenucci, M.A. Santos, L. Romani, M.G. Netea, and D. Cavalieri, Fungal chitin induces trained immunity in human monocytes during cross-talk of the host with Saccharomyces cerevisiae. J Biol Chem (2016).

[34] K. Suzuki, Y. Okawa, K. Hashimoto, S. Suzuki, and M. Suzuki, Protecting effect of chitin and chitosan on experimentally induced murine candidiasis. Microbiol Immunol 28 (1984) 903-12.

[35] Y. Okawa, K. Suzuki, M. Kobayashi, M. Asagi, K. Sakai, S. Suzuki, and M. Suzuki, Protective effect of acidic mannan fraction of bakers' yeast on experimental candidiasis in mice. Microbiol Immunol 30 (1986) 957-67.

[36] N.R. Di Luzio, and D.L. Williams, Glucan-induced modification of the increased susceptibility of cyclophosphamide-treated mice to Staphylococcus aureus infection. Cancer Immunol Immunother 6 (1979).

[37] B.M. Buddle, H.D. Pulford, and M. Ralston, Protective effect of glucan against experimentally induced staphylococcal mastitis in ewes. Vet Microbiol 16 (1988) 6776.

[38] G. Hetland, M. Lovik, and H.G. Wiker, Protective effect of beta-glucan against mycobacterium bovis, BCG infection in BALB/c mice. Scand J Immunol 47 (1998) 548-53.

[39] J.A. Cook, T.W. Holbrook, and W.J. Dougherty, Protective effect of glucan against visceral leishmaniasis in hamsters. Infect Immun 37 (1982) 1261-9.

[40] A.S. Al Tuwaijri, A.A. Mahmoud, I.A. Al Mofleh, and S.A. Al Khuwaitir, Effect of glucan on Leishmania major infection in BALB/c mice. J Med Microbiol 23 (1987) 363-5.

[41] D.L. Williams, and N.R. Di Luzio, Glucan-induced modification of murine viral hepatitis. Science 208 (1980) 67-9.

[42] K. Jung, Y. Ha, S.K. Ha, D.U. Han, D.W. Kim, W.K. Moon, and C. Chae, Antiviral effect of Saccharomyces cerevisiae beta-glucan to swine influenza virus by increased production of interferon-gamma and nitric oxide. Journal of veterinary medicine 51 (2004) 72-6.

[43] J.A. Reynolds, M.D. Kastello, D.G. Harrington, C.L. Crabbs, C.J. Peters, J.V. Jemski, G.H. Scott, and N.R. Di Luzio, Glucan-induced enhancement of host resistance to selected infectious diseases. Infect Immun 30 (1980) 51-7.

[44] I. Lavi, D. Levinson, I. Peri, L. Nimri, Y. Hadar, and B. Schwartz, Orally administered glucans from the edible mushroom Pleurotus pulmonarius reduce acute inflammation in dextran sulfate sodium-induced experimental colitis. Br J Nutr 103 (2010) 393-402. 
[45] F. Han, H. Fan, M. Yao, S. Yang, and J. Han, Oral administration of yeast $\beta$-glucan ameliorates inflammation and intestinal barrier in dextran sodium sulfate-induced acute colitis. Journal of Functional Foods 35 (2017) 115-126.

[46] S.E. Heinsbroek, D.L. Williams, O. Welting, S.L. Meijer, S. Gordon, and W.J. de Jonge, Orally delivered beta-glucans aggravate dextran sulfate sodium (DSS)-induced intestinal inflammation. Nutr Res 35 (2015) 1106-12.

[47] P.J. Rice, B.E. Lockhart, L.A. Barker, E.L. Adams, H.E. Ensley, and D.L. Williams, Pharmacokinetics of fungal (1-3)-beta-D-glucans following intravenous administration in rats. Int Immunopharmacol 4 (2004) 1209-15.

[48] H. Yoshitomi, N. Sakaguchi, K. Kobayashi, G.D. Brown, T. Tagami, T. Sakihama, K. Hirota, S. Tanaka, T. Nomura, I. Miki, S. Gordon, S. Akira, T. Nakamura, and S. Sakaguchi, A role for fungal \{beta\}-glucans and their receptor Dectin-1 in the induction of autoimmune arthritis in genetically susceptible mice. J Exp Med 201 (2005) 949-60.

[49] M. Ruutu, G. Thomas, R. Steck, M.A. Degli-Esposti, M.S. Zinkernagel, K. Alexander, J. Velasco, G. Strutton, A. Tran, H. Benham, L. Rehaume, R.J. Wilson, K. Kikly, J. Davies, A.R. Pettit, M.A. Brown, M.A. McGuckin, and R. Thomas, beta-glucan triggers spondylarthritis and Crohn's disease-like ileitis in SKG mice. Arthritis Rheum 64 (2012) 2211-22.

[50] S. Bekkering, J. Quintin, L.A. Joosten, J.W. van der Meer, M.G. Netea, and N.P. Riksen, Oxidized low-density lipoprotein induces long-term proinflammatory cytokine production and foam cell formation via epigenetic reprogramming of monocytes. Arterioscler Thromb Vasc Biol 34 (2014) 1731-8.

[51] J. Chen, and K. Raymond, Beta-glucans in the treatment of diabetes and associated cardiovascular risks. Vasc Health Risk Manag 4 (2008) 1265-72.

[52] G.C. Chan, W.K. Chan, and D.M. Sze, The effects of beta-glucan on human immune and cancer cells. J Hematol Oncol 2 (2009) 25.

[53] A.T. Borchers, J.S. Stern, R.M. Hackman, C.L. Keen, and M.E. Gershwin, Mushrooms, tumors, and immunity. Proc Soc Exp Biol Med 221 (1999) 281-93.

[54] M. Zhang, J.A. Kim, and A.Y. Huang, Optimizing Tumor Microenvironment for Cancer Immunotherapy: beta-Glucan-Based Nanoparticles. Frontiers in immunology 9 (2018) 341.

[55] G.D. Ross, V. Vetvicka, J. Yan, Y. Xia, and J. Vetvickova, Therapeutic intervention with complement and beta-glucan in cancer. Immunopharmacology 42 (1999) 61-74.

[56] V. Vetvicka, B.P. Thornton, and G.D. Ross, Soluble beta-glucan polysaccharide binding to the lectin site of neutrophil or natural killer cell complement receptor type 3 (CD11b/CD18) generates a primed state of the receptor capable of mediating cytotoxicity of iC3b-opsonized target cells. J Clin Invest 98 (1996) 50-61.

[57] V. Vetvicka, and J. Vetvickova, Combination of glucan, resveratrol and vitamin C demonstrates strong anti-tumor potential. Anticancer Res 32 (2012) 81-7.

[58] F. Hong, R.D. Hansen, J. Yan, D.J. Allendorf, J.T. Baran, G.R. Ostroff, and G.D. Ross, Beta-glucan functions as an adjuvant for monoclonal antibody immunotherapy by recruiting tumoricidal granulocytes as killer cells. Cancer research 63 (2003) 9023-31.

[59] F. Hong, J. Yan, J.T. Baran, D.J. Allendorf, R.D. Hansen, G.R. Ostroff, P.X. Xing, N.K. Cheung, and G.D. Ross, Mechanism by which orally administered beta-1,3-glucans enhance the tumoricidal activity of antitumor monoclonal antibodies in murine tumor models. J Immunol 173 (2004) 797-806.

[60] T.J. Yoon, T.J. Kim, H. Lee, K.S. Shin, Y.P. Yun, W.K. Moon, D.W. Kim, and K.H. Lee, Anti-tumor metastatic activity of beta-glucan purified from mutated Saccharomyces cerevisiae. Int Immunopharmacol 8 (2008) 36-42. 
[61] N. Sakaguchi, T. Takahashi, H. Hata, T. Nomura, T. Tagami, S. Yamazaki, T. Sakihama, T. Matsutani, I. Negishi, S. Nakatsuru, and S. Sakaguchi, Altered thymic T-cell selection due to a mutation of the ZAP-70 gene causes autoimmune arthritis in mice. Nature 426 (2003) 454-60.

[62] A. Bedirli, M. Kerem, H. Pasaoglu, N. Akyurek, T. Tezcaner, S. Elbeg, L. Memis, and O. Sakrak, Beta-glucan attenuates inflammatory cytokine release and prevents acute lung injury in an experimental model of sepsis. Shock 27 (2007) 397-401.

[63] C.T. Newsome, B. LeBlanc, A. Ayala, and J. Reichner, The effects of beta-glucan treatment on endotoxin and sepsis-induced cytokine production. FASEB J 23 (2009) supplement.

[64] C.T. Newsome, E. Flores, A. Ayala, S. Gregory, and J.S. Reichner, Improved antimicrobial host defense in mice following poly-(1,6)-beta-D-glucopyranosyl-(1,3)-beta-Dglucopyranose glucan treatment by a gender-dependent immune mechanism. Clinical and vaccine immunology : CVI 18 (2011) 2043-9.

[65] Z. Mirza, E.R. Soto, F. Dikengil, S.M. Levitz, and G.R. Ostroff, Beta-Glucan Particles as Vaccine Adjuvant Carriers. Methods Mol Biol 1625 (2017) 143-157.

[66] N.N. Acar, Ü. Noyan, L. Kuru, T. Kadir, and B. Kuru, Adjunctive Systemic Use of BetaGlucan in the Nonsurgical Treatment of Chronic Periodontitis 2012. 


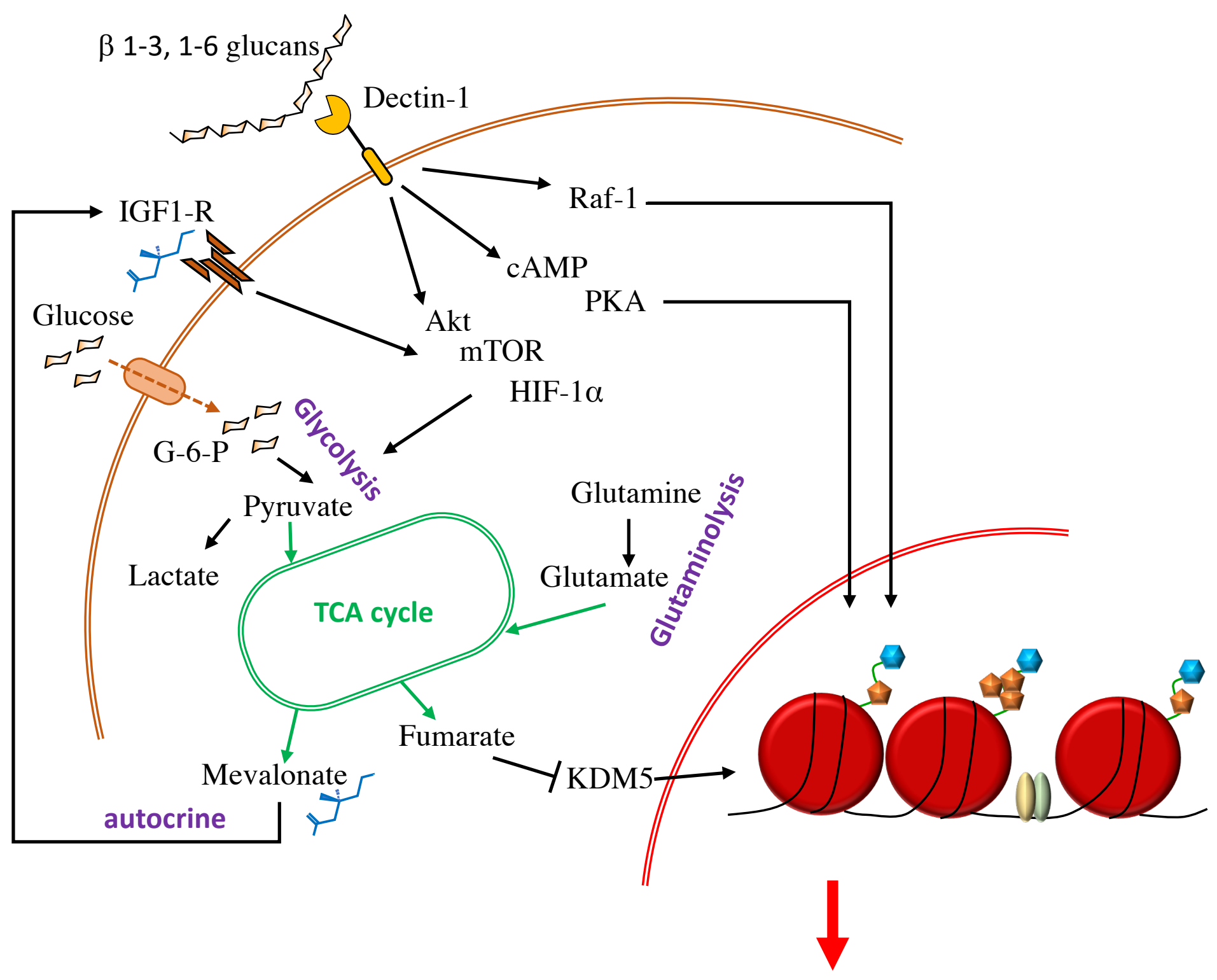

Cytokines/Inflammation/Protection against infection 


\begin{tabular}{|c|c|}
\hline $\begin{array}{l}\uparrow \text { LSKs } \\
\uparrow \mathrm{MPP3}=\text { MPP4 } \\
=\text { LT-HSCs } \downarrow \text { ST-HSCs }\end{array}$ & $24 \mathrm{~h}$ \\
\hline $\begin{array}{l}\uparrow \text { LSKs } \\
\uparrow \text { MPP3 } \downarrow \text { MPP4 } \\
\uparrow \text { LT-HSCS }\end{array}$ & $7 d$ \\
\hline $\begin{array}{l}=\text { LSKs } \\
=\text { LT-HSCs }\end{array}$ & $28 \mathrm{~d}$ \\
\hline
\end{tabular}

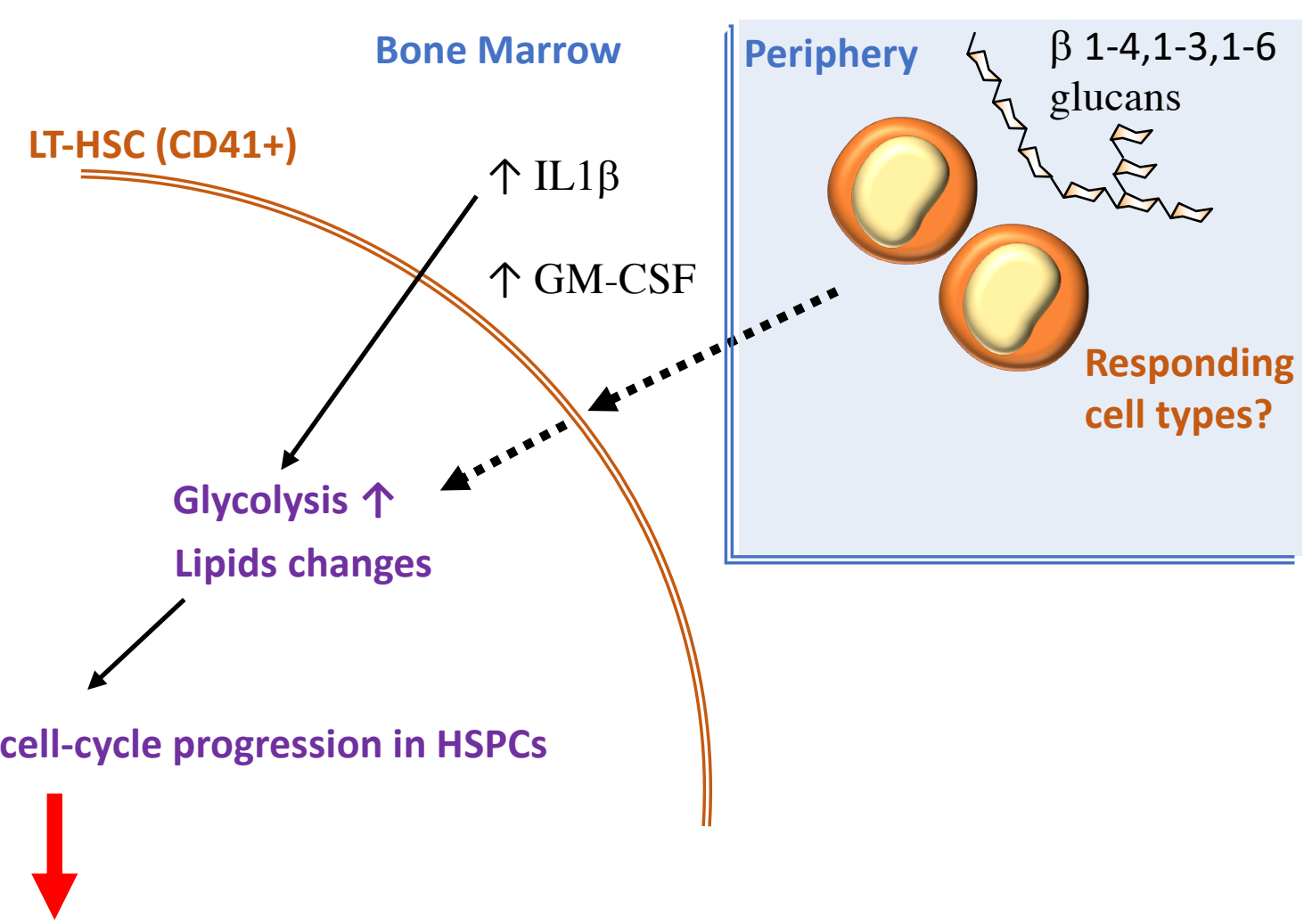

Improved resistance to chemotherapy

Protective response to DNA damage 